Document downloaded from:

http://hdl.handle.net/10251/65535

This paper must be cited as:

Sendra, S.; Lloret, J.; Jimenez, JM.; Parra, L. (2015). Underwater Acoustic Modems. IEEE Sensors Journal. 16(11):4063-4071. doi:10.1109/JSEN.2015.2434890.

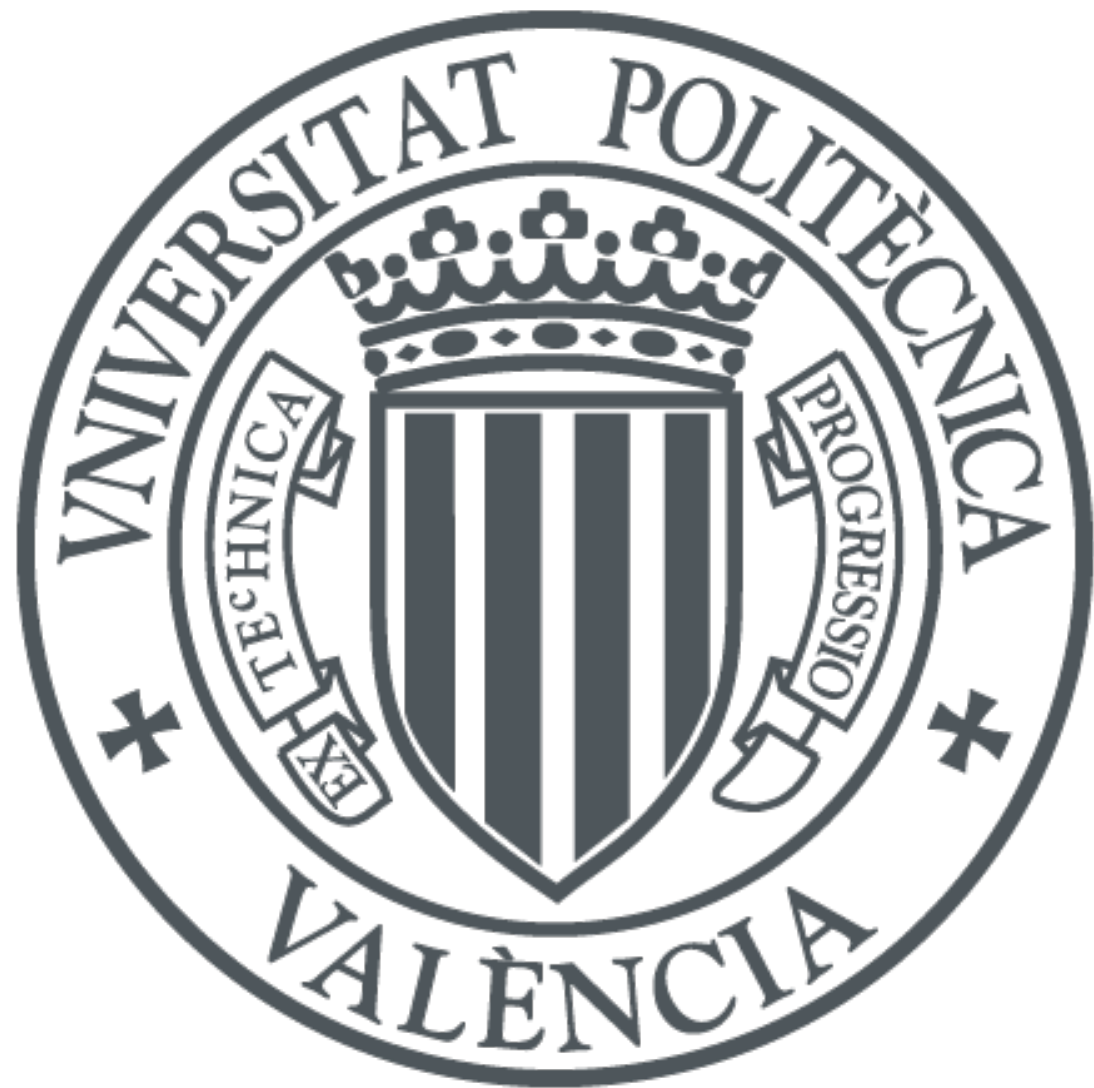

The final publication is available at

http://dx.doi.org/10.1109/JSEN.2015.2434890

Copyright Institute of Electrical and Electronics Engineers (IEEE)

Additional Information

(C) 2016 IEEE. Personal use of this material is permitted. Permission from IEEE must be obtained for all other uses, in any current or future media, including reprinting/republishing this material for advertising or promotional purposes, creating new collective works, for resale or redistribution to servers or lists, or reuse of any copyrighted component of this work in other works. 


\title{
Underwater Acoustic Modems
}

\author{
Sandra Sendra, Member IEEE, Jaime Lloret, Senior Member IEEE, Jose Miguel Jimenez, Lorena \\ Parra.
}

\begin{abstract}
Due to the growing interest using underwater acoustic networks, there are more and more research papers about underwater communications. These papers are mainly focused on deployments and studies about the constraints of the underwater medium. The underwater acoustic channel is highly variable and the signal transmission can change according to environmental factors such as the temperature, pressure or salinity of the water. For this reason, it is important to know how these devices are developed and the maximum distance and data transfer rates they can achieve. To this end, this paper presents an exhaustive study of existing underwater acoustic modems where their main features are highlighted. We also review the main features of their hardware. All presented proposals in the research literature are compared with commercial underwater acoustic modems. Finally, we analyze different programs and improvements of existing network simulators that are often used to simulate and estimate the behavior of underwater networks.
\end{abstract}

Index Terms-Underwater Acoustic Modem, Underwater Modems Deployment, Underwater Communication, Wireless Communication.

\section{INTRODUCTION}

$I_{w}^{\mathrm{N}}$ $\mathrm{N}$ recent years the scientific community has seen how wireless sensor networks have become more and more common. Different applications have been developed in order to bring revolutionary solutions to many issues such as surveillance and alarm systems of volcanoes activity [1], early detection of forest fires [2], air quality monitoring [3] and even home automation functionalities [4]. Although the main applications are developed for terrestrial environments, few years ago, the applications for underwater environments started to increase $[5,6]$. The main problems of underwater applications are the troubles deploying the sensors and the sensor maintenance. It is also quite complicated to apply energy harvesting and they usually have very high fabrication cost. Finally, it is complicated to achieve good performance in terms of data transfer rates because of the signal propagation difficulty in this dispersive medium [5]. In terrestrial applications, the communication between nodes is usually wireless and uses different technologies (mainly Wi-Fi, Zigbee or Bluetooth) in underwater applications the wireless communication becomes harder. Wireless technologies used in

J. Lloret is with the Universitat Politècnica de València, Camino de Vera s/n, 46022, Valencia (Spain) (corresponding author, phone: +34 609549043; fax: +34 962849313; e-mail: jlloret@dcom.upv.es).

S. Sendra, J.M. Jimenez, L. Parra are with the Universitat Politècnica de València, Camino de Vera $\mathrm{s} / \mathrm{n}$, 46022, Valencia (Spain) (e-mail: sansenco@posgrado.upv.es. jojiher@dcom.upv.es, loparbo@doctor.upv.es). terrestrial applications are not useful inside the water due to its composition. Another difference with the terrestrial networks is that sensors can be easily deployed in the underwater environment using 3-D space [7] and other sensor placement algorithms [8]. It has brought new researches focused on the optimization of mobility, coverage and connectivity [9, 10, $11]$.

Underwater sensor networks have many applications, as in terrestrial environments. However, underwater environment is far less known due to the hard conditions such as low temperature, changes in salinity or high pressure (which depends on the proximity to the seabed in deep sea). Sensor networks offer a feasible option to investigate those particular environments. There are many different applications in open sea where underwater wireless sensor networks (UWSNs) could be used. These are some of them [12, 13, 14]:

- Monitoring the effects of climate change

- Research about abyssal habitats

- Monitoring population changes on coral reefs

- Military purposes

- Search interesting areas for mining

- Water quality monitoring

- Prevention and alerts for disasters

- Fish farms monitoring

- Oceanic data collection

- Assisted navigation

- Offshore engineering monitoring

UWSNs offer useful solutions for coastal applications for water monitoring, fish farms control and seabed mining purposes [15, 16, 17]. The characteristics of underwater environments are very unstable. We can find big changes of temperature in very little difference of depth, different pressure, different levels of noise and even different water densities. The technology for underwater communications must be chosen carefully to ensure that the deployed systems are able to communicate for a long time. Underwater communications are more challenging than communications in terrestrial environments. The main issues of underwater acoustic communications are their low bandwidth, the propagation delay, high probability of bit errors and temporary loss of connectivity $[18,19]$. The radio frequency signals, so useful for terrestrial communications, are dramatically reduced in water; therefore the information only arrives to short distances [12]. That happens because unlike air, water is a conductive medium and causes great alterations on the electromagnetic radiation. Only at very low frequencies (3 to 
$30 \mathrm{kHz}$ ), the attenuation is lower enough to allow communicating two devices. However at these frequencies the obtained data rates are very low [6]. On the other hand, optical signals, which are a possible alternative in terrestrial applications, are not useful due to light scattering in the water medium [12]. Finally, optic fiber allows easily transmitting high data rates in underwater environment without any problem in the transmission. However, its limited flexibility makes it difficult to be used in several applications [9]. Optic fiber also has a high price that does not make it suitable for sensors communication [19]. In underwater environments, it is required high bandwidth and it is needed to achieve long distances. The apparition of acoustic modems has filled that gap on the existing technology. The underwater acoustic modems have to be energy efficient because the energy resources (even for energy harvesting) in underwater environments are limited. In addition, they should be cheap due to the high number of modems needed for monitoring huge areas [5]. Comparing with radio frequency signals, the hardware necessary for transmitting acoustic signals is cheaper [20]. Comparing the acoustic modems with Electro Magnetic (EM) modems, acoustic modems are much slower. In fact, the transmission of acoustic signal is approximately $1500 \mathrm{~m} / \mathrm{s}$ (five orders of magnitude slower than EM propagation) [21].

Two characteristics are required for acoustic communication [6]. On the one hand, it is needed a modulation phase (in the transmitter) and a demodulation phase (in the receiver) using a carrier wave to optimize the quantity of information sent and to decrease the effects of noise and interference. It is also needed a medium to transport that carrier wave. The water is a good medium to transmit the carrier wave with low noise rate. These facts make acoustic communication the most useful way to transfer data under the sea. However, acoustic modems have also some problems as transmission loss, propagation delay and Doppler Effect, refraction due to the variations of the temperature and pressure, multipath and even frequency attenuation [20]. The current developed acoustic modems can only support point-to-point, low-data-rate and delay-tolerant applications. In addition, the absence of accepted international standards has made the appearance of several proposals in a short period of time [18].

The rapid development of different underwater acoustic modem proposals makes very difficult to keep updated the information related to this topic. The related literature includes several surveys about underwater acoustic networks, about WSN architectures [10][11], about location techniques [13] and other general issues [18]. However there is no specific survey about acoustic modems, where authors focus the content on showing their main features.

In this paper, we present a depth study of existing underwater acoustic modems. We also review their main features. Along the document, we will see many proposals developed by researchers. In addition, these proposals are compared with commercial ones. Finally, we can see some of the main works related to software and network simulators for underwater networks.

The rest of the paper is structured as follows. Section II explains the architecture and main parts of an underwater acoustic modem. We explain the most interesting published proposals on underwater acoustic modems in Section III. This section also includes a comparative table of these proposals and the commercial devices in order to see their performance and maximum distances. Section IV presents the advances in software and simulators for underwater acoustic networks. Finally, conclusion and future work are shown in Section V.

\section{Design Of Underwater ACOUSTIC MODEMS}

In the recent years there is a huge research activity developing communications systems for underwater acoustic networks to mitigate its limitations of this environment such as the available bandwidth, distance, or channel latency [22] [23]. Like terrestrial networks, UWSN must present a flexible and scalable architecture to allow improvements and new additions, which are necessary for future research [24]. In essence, an underwater modem consists of:

1) A power unit, which has a battery and a set of DC/DC converters,

2) a processing unit, which usually consists of a small processor and memory (sometimes, it can be added as an external memory),

3) the physical hydrophone and loudspeaker,

4) circuitry (used to adapt the digital signals to the processor) and the analog to digital converter and the digital to analog converter to adapt changes between the medium and the electric circuit.

Figure 1 shows the block diagram of a typical underwater acoustic modem [25][26].

Because of the absence of wires, underwater oceanographic equipment is mainly dependent of batteries. With acoustic modems, data can be transmitted using wireless connections. However, most of the commercial modems use RS-232 or USB port to download some data stored in the device or to program it. Current acoustic modems consume too much power and thus, they are not suitable for long-term deployment. It is a main drawback that should be reduced.

Data transmission range and data transmission rates should be carefully studied. Thanks to the proprieties of the water, acoustic waves can be easily transmitted through it. Devices can communicate from less than a meter to hundreds of meters. However, parameters such as temperate, depth, water pressure can difficult the data transmission. More transmission power means more energy consumption. For this reason network development strategies must be focused on energy efficiency and should consider the number of wireless links, power requirements, and fabrication cost. In regards to data transmission rates, it is important to know that underwater environments are very dispersive mediums. This is the main reason because we can work from a few bps to hundreds of bps. Obviously, its value will be inversely proportional to the distance between devices. 


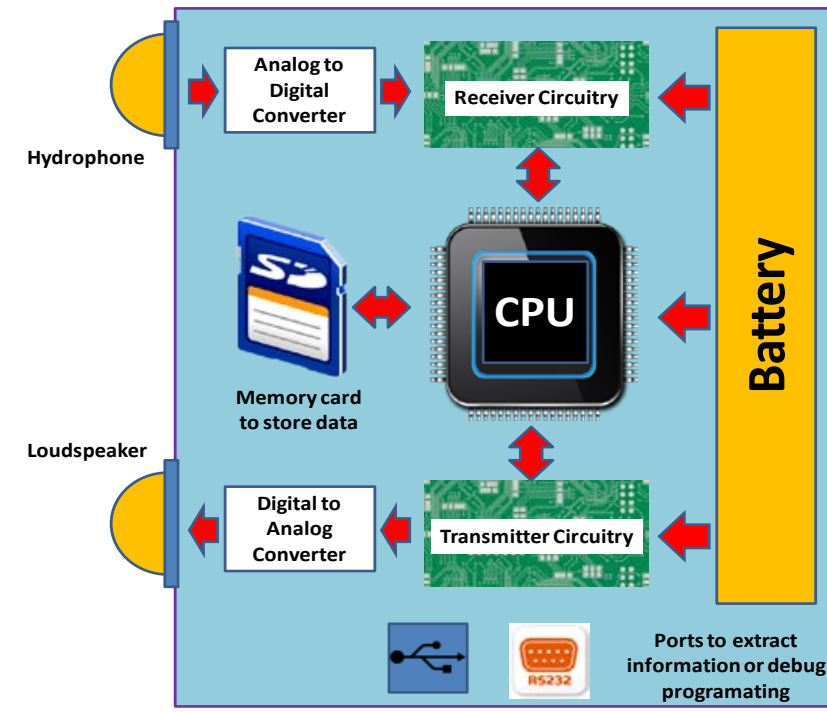

Fig. 1. Block diagram of an underwater acoustic modem.

Finally, algae or barnacles building up on a transducer can block the modem's signal, so data may be corrupted. For this reason, it is very important to have a suitable and waterproof packaging that should be resistant to biofowling.

\section{PRACTiCAl DEPLOYMENTS}

This section shows some interesting underwater acoustic modems developed in the last decade. This section also shows a summary table where these practical deployments are compared with commercial devices.

A. Sanchez et al. present in [5] an acoustic modem characterized by its low cost and its ultra-low energy consumption. Firstly, Authors describe the modem architecture. They used an 8-bit microcontroller and they added add to it some external analog components. Authors focused the work on the transmission and reception of an acoustic signal. They used a commercial device based on the use of piezoelectric materials, usually used for sonar. In order to guaranty the system performance and keep low power consumption, different modulation algorithms were compared. Finally authors choose Coherent-FSK algorithm. Proposed modems are able to send information at 1 kbps. The energy consumption and the price of their proposal are similar to the typical motes for terrestrial applications. The energy consumption when the modem is in standby mode is $3 \mu \mathrm{W}$. It consumes $24 \mathrm{~mW}$ in reception mode and $12 \mathrm{~mW}$ in transmission mode (the lowest energy consumption for acoustic modems until that date). Their proposal was tested in different scenarios: in a water tank, inside a port and finally in open sea. Authors measured the goodness of the transmission with the packet transmission range. The maximum distance reached in those tests was $100 \mathrm{~m}$.

In [19], N. Farr et al. showed the development of opticalbased underwater communication system. The developed system is able to complement current acoustic systems. The use of an optical-based model in combination with an acousticbased one can offer higher data rates and lower latency than the one obtained only with acoustic-based modem. Authors proposed to use this system for the communication between autonomous underwater vehicles (AUVs). They performed several tests in real environments. First test was carried out with an AUV in deep water. The emitter, positioned on the seabed, sent a signal to the AUV (where the receiver was placed), which was moving away from the emitter. The aim of the test was to find out the maximum distance between emitter and receiver without signal errors. The data rate of the emitter was 1 Mbps. The maximum distance with error free transmission recorded in the test was $100 \mathrm{~m}$. The second test was aimed to evaluate the viability of the proposed system for controlling underwater vehicles in real time. The optical-based modem was used to send video capture in real time to a receiver separated by $15 \mathrm{~m}$. The results show that the video signals arrived in real time without errors.

In [27], A. Sanchez et al. present the description of physical layer of an acoustic-based underwater modem with ultra-low power consumption and low fabrication cost. It also has no maintenance needs and long life-time. The modem is called ITACA. It includes an asynchronous wake-up system, which has low power consumption and is based on a low-cost off-theshelf RFID peripheral integrated circuit. The system has an energy consumption of $8.1 \mu \mathrm{W}$ when it is in stand-by mode. Their proposal also has clear channel assessment (CCA) to support CSMA-based medium access control (MAC) layer protocols. As modulation, authors propose the use of coherentFSK algorithm. ITACA uses only $24 \mathrm{~mW}$ for receiving data. For transmission, the maximum distance reached is $240 \mathrm{~m}$ using $108 \mathrm{~mW}$ of power consumption. The modems operated at $85 \mathrm{kHz}$ and reached a data rate of $1 \mathrm{kbps}$. The presented system is similar to the one presented in [5]. The main novelty of this proposal is the use of RFID technology. RFID technology is used to recognize 8 or 16-bit patterns without MCU activation. The inclusion of CCA mechanism is experimentally tested and provides a reliability of $99.7 \%$.

A highly flexible acoustic modem called the Reconfigurable Modem (rModem) is presented in [28] by E. Multu et al. The system is based on a main board with a daughter card interface. The main board is an Altera Cyclone II FPGA. The daughter card is used for adding an amplifier phase. It also allows working with several transducers. Authors use a Teledyne AT-408 omnidirectional transducer that operates in the $9-14 \mathrm{kHz}$ band. Authors also present a software application based on the Simulink environment and the Real-Time Workshop toolbox from MathWorks. The user guide interface is used to control the rModem hardware, send and receive packets, and log events and data. The system was tested using four nodes with different roles in open sea to test the cooperative communications scheme. The experiment was conducted with the source node and the destination node connected to a player/recorder device, while the repeater node was an rModem outfitted with a transducer. Authors state that rModem provides high processing gain and big memory capacity, making easy the coding requirements.

$\mathrm{N}$. Nowsheen et al. describe in [29] the performance of an 
underwater acoustic modem. The modem presented is based on a Cyclone III EP3C25 FPGA and operates at high frequencies and high data-rate communications. It is also designed to be a low cost solution. The main difference between their proposal and the existing modems in that date is that their proposal was based on DSP processors. The high flexibility of the modem offers the possibility of changing several parameters. It also minimizes the cost of rework. That flexibility is given because all the processing tasks are done in the digital domain. The modem uses BPSK modulation because it is less sensitive to noise. For the demodulation process, it uses BPSK with Costas loop. Authors develop different simulations and test. One of them was performed in real environment (in a lake). They tested the performance transmitting from different points in the lake. The transmitter and receiver were deployed at $3 \mathrm{~m}$ deep. Authors evaluated the signal taking samples every 250 ns. The modems operated at $80 \mathrm{kbps}$ at $800 \mathrm{kHz}$ during the test and they reached a maximum distance with good communication up to $50 \mathrm{~m}$.

L. Badia et al. [30] presented a new practical implementation for joint sensor deployment, link scheduling and routing in small underwater sensor networks. They developed a mathematical model to reproduce a three dimensional space of the sea as well as the acoustic waves propagation and interference conditions at each receiver in underwater environment. Authors have validated the model using a UWM1000 LinkQuest underwater acoustic modem. The results show that under certain conditions this device can be used to transmit data, but it is not expected to work at distances greater than $350 \mathrm{~m}$.

I. Vasilescu et al. [31] described the hardware and designed and implemented in their laboratory to deploy an underwater sensor network. In their system, nodes communicate acoustically. Data are broadcasted using an acoustic modem developed by them. Authors describe the hardware details of the underwater sensor node, the communication and the networking protocols. Their experiments show that this system is useful for water applications in shallow waters at depths lower than $100 \mathrm{~m}$, but sensors can operate at greater depths by replacing the acrylic enclosure with a glass or titanium enclosure. The system is intended to be used to provide automated data collection for marine biology applications for understanding and modeling coral reefs.

I. Vasilestu et al. [32] also presented a platform, composed by both parts hardware and software for underwater sensor networks, which can be used for long-term monitoring of coral reefs and fisheries. The sensor network consists of a set of static and mobile underwater sensor nodes. These nodes have a variety of sensing capabilities, including cameras, water temperature, and pressure. Authors reported their first prototype which was developed and really deployed. They described the optical and acoustic network protocols and presented the experimental results including data collected in a pool, in rivers, and in the ocean. The test bench also included some studies of mobility for data mulling. Results demonstrated that sensor networks are feasible for underwater uses and that data mulling provides an effective way to collect, store, and retrieve large volumes of data over long periods of time. They argue that data mulling provides a significant power advantage over an acoustic communication network with multi-hop routing.

B. Benson et al. [25] described the major design components of their low-cost prototype of an underwater acoustic modem intended for short-range communication, for applications with low data rate requirements. The digital hardware platform has been tested and results show that it can support data rates of up to 200 bps with bit error rate at a low signal to noise ratio (SNR) of $10 \mathrm{~dB}$. Authors developed a power supply board composed by a battery pack and a watertight housing that can withstand pressures at depth of up to $100 \mathrm{~m}$. to be able to test the modem in the open ocean in order to assess its performance. Initial in-water tests of the transducer and analog transceiver in Mission Bay, CA. USA, indicated that their design is capable of detecting a signal at distances greater than $350 \mathrm{~m}$.

L. Wu et al. [33] presented the design of a modem, that provides reliable and energy efficient communication in underwater sensor networks. Their work was supported with results from simulations and experiments. These experiments and sea tests quantified the benefits given by different modulations, types of channel estimation, and symbol synchronization. Results show that rate adaptation can lead to substantial energy savings while ensuring reliable communication.

J. Wills et al. [34] described the design of a low-power acoustic modem for underwater sensor networks. The rationale behind of their design is to support large-scale, long-lived, and dense sensor networks powered by batteries. Finally, authors presented some preliminary results in terms of transmitted waveform. The results were very promising.

S. Singh et al. [35] presented WHOI Micro-Modem that is a compact, low-power acoustic transceiver that can provide both acoustic telemetry and navigation. Its size and versatility make it ideal to be integrated in AUVs. The modem supports the use of both broadband and narrowband transponders for long baseline navigation systems. It has a modem-to-modem ranging capability, and can be configured to provide synchronous one way ranging, when it is integrated with a precision clock. Authors also gave a summary of the different navigation systems supported by the Micro-modem and presented the results from field tests conducted on the SeaBED AUV in Greece, the Bluefin AUV, and whale localizations in the Stellwagen Bank Marine Sanctuary.

J. Heidemann et al. [36] presented their research in underwater sensor networks. They highlighted potential applications to off-shore oil fields for seismic monitoring, equipment monitoring, and underwater robotics. They also identified research directions in short-range acoustic communications, MAC protocols, time synchronization, and localization protocols for high latency acoustic networks, long- 
duration network sleeping, and application-level data scheduling. Finally, authors summarized some of the main

applications that this kind of networks can improve.

TABLE I

COMPARISON OF COMMERCIAL DEVICES AND DEVELOPED DEVICES

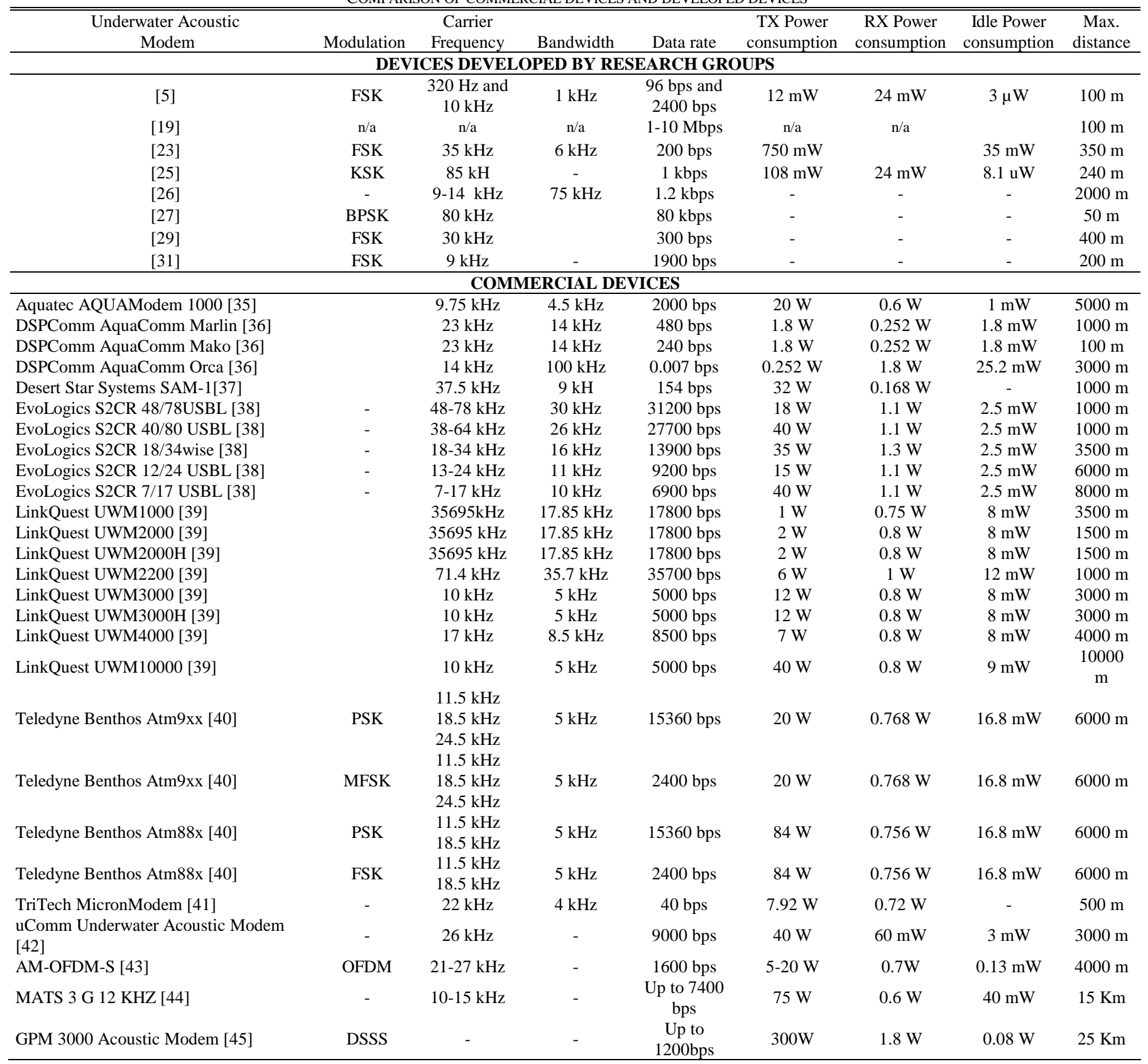

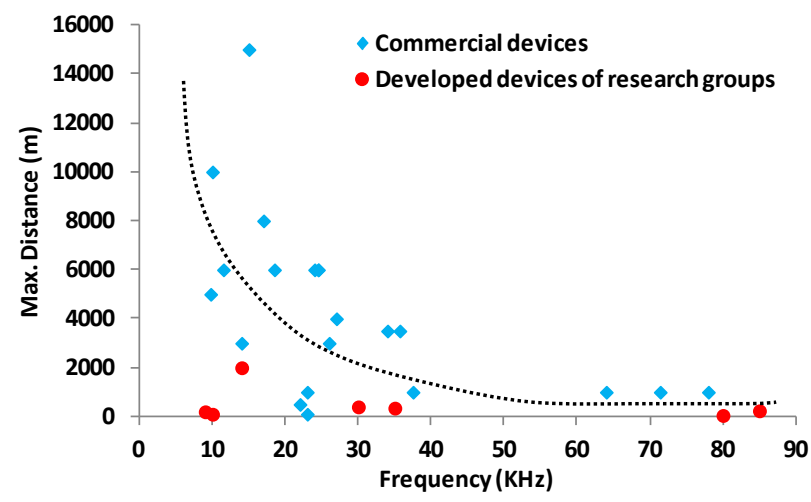

Fig. 2. Relationship between working frequency and maximum distance
Table I shows the main features of the proposed deployments. In addition, this table contains some of the most important commercial underwater acoustic modems we can find in the market. These comparative is performed in terms of energy consumption, maximum distances, bandwidth and data transfer rates.

Figure 2 shows the relationship between the working frequency and the maximum distance achieved by each device. Commercial devices are displayed in blue while devices developed by research groups are shown in red. The first observation is that this relationship is similar to an exponential behavior, although a high accentuated dispersion is shown. 
This could be mainly due to the type of modulation used, and even, it is possible that the type of transducer used affects these parameters. It is also easy to see that commercial devices have greater distances (See Figure 2) but its energy consumption is, in all cases, much higher (See Table I). Finally, we present some studies performed in recent years where authors compare several commercial modems which are shown in Table I.

The energy saving through the use of wakeup solutions is not new but it was never applied to acoustic modems. F. Harris III et al. [48] presented a comparative study of two techniques for idle-time power management to optimize the energy efficiency in underwater acoustic modems. Authors stated that in UWSNs the traffic expected is less than one packet/node/hour. Authors performed several simulations to test the energy consumption of different protocols used by underwater acoustic modems. They used four different protocols; the first one did not use any sleep/wakeup mode. The rest of them included it. The second protocol was an optimal sleep protocol. The third was their proposal and the last one was STEM (sleep cycling protocol). In order to evaluate the protocols authors compared the total energy consumption and the death time of the first node. Their results show that the wakeup mode saved more energy than sleep cycling.

D. L. Codiga et al. [49] presented the results of underwater acoustic modems used for wireless real-time delivery of oceanographic measurements from a distributed array of subsurface instruments in coastal waters. The tested network consisted of sensor nodes, repeater nodes, gateway nodes, and a shore-based control center. The test bench was performed in the inner continental shelf off Montauk Point, New York, and Block Island, Rhode Island. They used a U.S. Coast Guard navigation buoy equipped as a gateway. The tests we performed varying several conditions such as the sound speed, water depths $(\sim 25-50 \mathrm{~m})$, and seasons. Long-term average rates of successful transmissions fell to about $50 \%$ at a range of 3-4 km in the shallow-water acoustic channel. Modem range degrades with increased winds. The $\mathrm{r}$ system had a $42 \%$ success rate at $5 \mathrm{~km}$ range for data packets of a few hundred bytes in size transmitted every $40 \mathrm{~min}$ during a two weeks experiment. Authors concluded that real-time communications, facilitated by deploying acoustic modems, have practical benefits for real-time data delivery.

R. M. Eustice et al. [50] showed experimental results of the development and deployment of a synchronous-clock acoustic navigation system suitable for the simultaneous navigation of multiple AUVs. Their navigation system is composed of an acoustic modem-based communication/navigation system that allows broadcasting the onboard navigational data form a source node for all passive receiving nodes. These data can be used to decode the data packet for obtaining a one-way trip time pseudo-range measurement and ephemeris data. Authors also present results for two different field experiments using a two-node configuration consisting of a global positioning system (GPS) and a surface ship that acts as a global navigation aid to the Doppler-aided of the AUV navigation.

E. R. B. Marques et al. [51] presented a framework to communicate and control an AUV. This work is an important step towards the control of heterogeneous vehicle systems through dynamic environments, combining the integration of operators in the planning and control loops.

A. Bahr et al. [52] described an algorithm designed for distributed acoustic navigation for AUVs and presented the experimental validation in a sequence of test bench using a variety of autonomous marine platforms. The algorithm is particularly well suited for underwater applications where the communication bandwidth is severely limited. Their work seeks to create a fully mobile network of AUVs that perform data exchange with another device to achieve cooperative positioning for extended duration missions over large areas.

\section{SOFTWARE AND SIMULATORS FOR UNDERWATER ACOUSTIC NETWORKS}

In a way, the underwater environment and how it behaves against a range of situations, is unknown to humans. It is also difficult to predict what will happen to the transmitted signals and the performance of the developed system. For this reason, more and more new simulators and mathematical models are being developed. The novelty is that they are able to give a point of realism to the pre-launch test. A. F. Harris and M. Zorzi presented in [53] the design and implementation of an interface and channel model for underwater acoustic networks based on the ns2 network simulator. In that case, because ns2 usually assumes a fixed bandwidth used by layers higher than the propagation and channel model, authors provided new modules for each network layer, allowing protocol developers to concentrate efforts on the higher layers of the network protocol stack.

A new framework for simulating underwater communication protocols is presented in [54], by C. Petrioli et al., where ns2 is used as the network simulator. Authors propose an efficient architecture that allows anyone to use the same code to perform simulations of acoustic underwater modems in order to test their protocols. The developed code can be stored in small portable devices. This improvement makes possible to embed this code in the acoustic modem in order to perform real tests. To test the feasibility of proposed architecture, authors performed different test benches with several micromodems. They obtained promising results with few milliseconds of delays and an extra overhead of just one extra byte per packet. Their framework seems to be a very useful tool for designing and testing protocols.

F. Guerra et al. described in [55] a network simulator for underwater networks which explicitly incorporates the detailed propagation behavior combining the well-known ns2 network simulator and MIRACLE simulators with the Bellhop ray tracing tool. Ray-tracing provides accurate emulation of sound propagation. Flexible programming at all levels of the protocol allows presenting more realistic reproduction of underwater 
behavior estimations. This work also present a comparative study of MAC protocols for an underwater network where protocols such as ALOHA, Tone-Lohi and DACAP are generally used. Their work also provides a discussion of the relationship between the amount of coordination and the final network performance. The results compare the transmission coordination approach chosen by each protocol, and show when it is better to rely on random access, as opposed to lose or tight coordination.

Finally, P. Xie et al. presented in [56] a network simulator called Aqua-Sim, for simulating underwater sensor networks. Aqua-Sim can simulate the collision behaviors in long delay acoustic networks and the signal attenuation that underwater acoustic channels can suffer. In addition, this simulator is able to simulate three-dimensional network deployment and authors added several protocol definitions to make it more accurate. Finally, authors demonstrated the power, its high fidelity, and the flexibility of Aqua-Sim through several case studies, analyzing parameters such as energy consumption and packet deliver ratio among others.

As we can see, there are very few works focused on the deployment of accurate simulators for underwater networks. In addition all of them are based on the ns2 network simulator. In these cases, authors tried to add new features and protocol definitions to the basic ns2 structure.

\section{CONCLUSION AND FUTURE WORK}

In recent years, there have been lots of underwater devices such as sensors and underwater modems. Such devices have very good acceptance in applications such as monitoring of marine environments, animal tracking and military applications. Therefore, in this paper we have studied the types of devices we can currently find on the market and the main quality requirements they should present. Devices developed and tested by research groups have been compared with commercial devices. As Table I shows commercial devices can reach longer distances. However, energy consumption is also much higher.

Finally, we analyzed the investigations made in regards to software and simulators of underwater acoustic networks. As we have seen, researchers have mainly based their improvements on the ns2 Network simulator, adding and modifying some parts to program features and protocols definitions to make the simulation results more approximate to actual results.

As future work, we would like to perform a new study for comparing the underwater acoustic communications and the devices used with this technology and underwater communications based on electromagnetic waves [57][58].

\section{ACKNOWLEDGMENT}

This work has been partially supported by the "Ministerio de Ciencia e Innovación", through the "Plan Nacional de $\mathrm{I}+\mathrm{D}+\mathrm{i}$ 2008-2011" in the "Subprograma de Proyectos de Investigación Fundamental”, project TEC2011-27516.

\section{REFERENCES}

[1] R. Tan, G. Xing, J. Chen, W. Z. Song, and R. Huang, "Fusion-based volcanic earthquake detection and timing in wireless sensor networks". ACM Transactions on Sensor Networks, 2013, vol. 9, no 2, pp. 17.

[2] J. Lloret, M. Garcia, D. Bri, and S. Sendra, "A wireless sensor network deployment for rural and forest fire detection and verification”. Sensors, 2009, vol. 9, no 11, p. 8722-8747.

[3] A. R. Al-Ali, I. Zualkernan and, F. Aloul, "A mobile GPRS-sensors array for air pollution monitoring”. Sensors Journal, IEEE, 2010, vol. 10, no 10, pp. 1666-1671.

[4] T. O. Arcas, R. Vilches, J. A. Segura and, M. Bajet, "Pervasive sensors network for wellness based-on Raspberry Pi". Network Protocols and Algorithms, 2014, vol. 6, no 3, pp. 1-17.

[5] A. Sanchez, S. Blanc, P. Yuste and, J. J. Serrano, "A low cost and high efficient acoustic modem for underwater sensor networks". In Procedings of IEEE-Spain OCEANS, 2011, 6 - 9 June, 2011, Santander (Spain), pp. 1-10.

[6] P. Lacovara, "High-bandwidth underwater communications". Marine Technology Society Journal, 2008, vol. 42, no 1, p. 93-102.

[7] S. Matsumae, "Energy-Efficient Cell Partition of 3D Space for Sensor Networks with Location Information", Network Protocols and Algorithms, Vol 1, No 2 (2009). Pp. 85-98

[8] A. Pal, "Localization Algorithms in Wireless Sensor Networks: Current Approaches and Future Challenges", Network Protocols and Algorithms, Vol 2, No 1 (2010). pp. 45-73

[9] K. Akkaya and, A. Newell, "Self-deployment of sensors for maximized coverage in underwater acoustic sensor networks". Computer Communications, 2009, vol. 32, no 7, pp. 1233-1244.

[10] J. Lloret, "Underwater sensor nodes and networks", Sensors, 2013, Vol. 13, Issue 9, pp. 11782-11796.

[11] A. Hawbani, X. Wang, N. Husaini, S. Karmoshi, "Grid Coverage Algorithm \& Analyzing for wireless sensor networks", Network Protocols and Algorithms, Vol 6, No 4 (2014). pp. 1-18

[12] M. Erol-Kantarci, H. T. Mouftah and, S. Oktug, "A survey of architectures and localization techniques for underwater acoustic sensor networks”. Communications Surveys \& Tutorials, IEEE, 2011, vol. 13, no 3, pp. 487-502.

[13] H. P. Tan, R. Diamant, W. K. Seah and, M. Waldmeyer, "A survey of techniques and challenges in underwater localization”. Ocean Engineering, 2011, vol. 38, no 14, pp. 1663-1676

[14] I. F. Akyildiz, D. Pompili and, T. Melodia, "Underwater acoustic sensor networks: research challenges”. Ad hoc networks, 2005, vol. 3, no 3, pp. 257-279.

[15] M. Garcia, S. Sendra, G. Lloret, J. Lloret, "Monitoring and control sensor system for fish feeding in marine fish farms", IET communications, Vol. 5, Issue 12, pp. 1682-1690. 2011

[16] J. Lloret, S. Sendra, M. Garcia, G. Lloret, "Group-based underwater wireless sensor network for marine fish farms", IEEE GLOBECOM Workshops 2011. pp. 115-119. 2011

[17] J. Lloret, M. Garcia, S. Sendra, G. Lloret, "An underwater wireless group-based sensor network for marine fish farms sustainability monitoring”, Telecommunication Systems, 1-18. 2014. Doi: 10.1007/s11235-014-9922-3

[18] Z. Jiang, "Underwater acoustic networks-issues and solutions". International journal of intelligent control and systems, 2008, vol. 13, no 3, pp. 152-161.

[19] N. Farr, A. Bowen, J. Ware, C. Pontbriand and, M. Tivey, "An integrated, underwater optical/acoustic communications system”. In Procedings of IEEE-Sydney OCEANS 2010, 24 - 27 May, 2010, Sidney (Australia), pp. 1-6

[20] K. D. Frampton, "Acoustic self-localization in a distributed sensor network”. Sensors Journal, IEEE, 2006, vol. 6, no 1, pp. 166-172.

[21] N. Nowsheen, C. Benson and, M. Frater, "A high data-rate, softwaredefined underwater acoustic modem". In Procedings of IEEE-Sydney OCEANS 2010, 24 - 27 May, 2010, Sidney (Australia), pp. 1-5.

[22] M. Stojanovic, "Underwater acoustic communications: Design considerations on the physical layer". WONS 2008, pp. 1-10, Jan. 2008, Garmisch-Partenkirchen (Germany)

[23] M. Garcia, S. Sendra, M. Atenas, and J.Lloret, "Underwater Wireless Ad-hoc Networks: a Survey”, Ch. Book 14, Mobile Ad hoc Networks: Current Status and Future Trends. 2011, CRC Press, Taylor and Francis, pp. 379-411 
[24] J. H. Cui, J. Kong, M., Gerla and, S. Zhou, "The challenges of building mobile underwater wireless networks for aquatic applications", Network, IEEE, , vol. 20, issue 3, pp. 12-18, May-June 2006

[25] B. Benson, L. Ying, B. Faunce, K. Domond, D. Kimball, C. Schurgers, and R. Kastner, "Design of a Low-Cost Underwater Acoustic Modem", Embedded Systems Letters, IEEE, vol. 2, issue 3, pp 58-61, May 2010

[26] S. Sendra, "Deployment of Efficient Wireless Sensor Nodes for Monitoring in Rural, Indoor and Underwater Environments". 2013. Editorial Universitat Politècnica de València. Available at: http://riunet.upv.es/handle/10251/32279

[27] A. Sánchez, S. Blanc, P. Yuste, A. Perles, and J. J. Serrano, An ultralow power and flexible acoustic modem design to develop energyefficient underwater sensor networks. Sensors, 2012, vol. 12, no 6, pp. 6837-6856.

[28] E. M. Sözer and, M. Stojanovic, "Reconfigurable acoustic modem for underwater sensor networks". In Proceedings of the 1st ACM international workshop on Underwater networks. ACM, $29-29$ September 2006, Los Angeles (USA), pp. 101-104.

[29] N. Nowsheen, C. Benson and, M. Frater, "A high data-rate, softwaredefined underwater acoustic modem". In Procedings of IEEE-Sydney OCEANS 2010, 24 - 27 May, 2010, Sidney (Australia), pp. 1-5.

[30] L. Badia, M. Mastrogiovanni, C. Petrioli, S. Stefanakos and, M. Zorzi, "An optimization framework for joint sensor deployment, link scheduling and routing in underwater sensor networks", ACM SIGMOBILE Mobile Computing and Communications Review, vol. 11, issue 4, pp. 44-56, Oct. 2007

[31] I. Vasilescu, C. Detweiler, D. Rus, "AquaNodes: an underwater sensor network", Proc. WuWNet '07, pp. 85-88, Sep. 2007, Montreal (Canada)

[32] I. Vasilescu, K. Kotay, D. Rus, M. Dunbabin, P. Corke, " Data collection, storage, and retrieval with an underwater sensor network", Proc. of SenSys'05, pp. 154-165, Nov. 2005, San Diego (USA)

[33] L. Wu, J. Trezzo, D. Mirza, P. Roberts, J. Jaffe, Y. Wang and, R. Kastner, "Designing an adaptive acoustic modem for underwater sensor networks", Embedded Systems Letters, IEEE, vol. 4, issue 1, pp. 1-4, March 2011

[34] J. Wills, W. Ye, J. Heidemann, "Low-power acoustic modem for dense underwater sensor networks", Proc. WUWNet '06, pp. 79-85, September 2006, Los Angeles (USA)

[35] S. Singh, M. Grund, B. Bingham, R. Eustice, H. Singh, L. Freitag, "Underwater acoustic navigation with the WHOI micro-modem", OCEANS 2006, pp. 1-4, Sept. 2006, Boston (USA)

[36] J. Heidemann, Y. Li, A. Syed, J. Wills, W. Ye, "Underwater sensor networking: Research challenges and potential applications", Procedings of the Technical Report ISI-TR-2005-603, USC/Information Sciences Institute, 2005

[37] Aquatec AQUAModem 1000 features. In aquatecgroup web site. Available http://www.aquatecgroup.com/images/datasheets/aquamodem1000.pdf [Last access: March 31, 2015]

[38] DSPComm devices features. In dspcomm web site. Available at: http://www.dspcomm.com/docs/Aquacomm_Information.PDF

[39] Desert Star Systems SAM-1 features. In desertstart web site. Available at: http://desertstar.com/product/sam-1/ [Last access: March 31, 2015]

[40] EvoLogics acoustic Modems features. In evologics web site. Available at: http://www.evologics.de/en/products/acoustics/index.html [Last access: March 31, 2015]

[41] LinkQuest underwater acoustic modems features. In link-quest web site. Available at: http://link-quest.com/html/models1.htm [Last access: March 31, 2015]

[42] Features of Acoustic Modems Teledyne Benthos. In f-e-t web site. Available at: http://www.f-et.com/images/uploads/Teledyne_Benthos_ATM-885_Modem_UDB9000_Deck_Unit_with_Hydrophone.pdf [Last access: March 31, 2015]
[43] TriTech MicronModem features. In tritech web site. Available at: http://www.tritech.co.uk/product/micron-data-modem [Last access:March 31, 2015]

[44] uComm Underwater Acoustic Modem features. In sonardyne web site. Available at: http://www.sonardyne.com/images/stories/datasheets/Sonardyne_8260_ uCOMM.pdf [Last access: March 31, 2015]

[45] AM-OFDM-S features. In squarespace web site. Available at: http://static1.squarespace.com/static/53f382fae4b05ea0cfc56833/t/5407 5f44e4b0f45128a7a5a0/1409769284704/AM-OFDM-S1.pdf [Last access: March 31, 2015]

[46] MATS 3 G 12 KHZ features. In sercel web site. Available at: http://www.sercel.com/products/Lists/ProductSpecification/Mats3G_spe cifications_Sercel_SP.pdf [Last access: March 31, 2015]

[47] GPM 3000 Acoustic Modem features. In l-3com web site. Available at: http://www2.l-

3com.com/oceania//pdfs/datasheets/GPM\%20300\%20Acoustic\%20Mo dem\%20Spec\%20Sheet\%20Rev\%201\%206.pdf [Last access: March 31, 2015]

[48] A. F. Harris, M. Stojanovic and, M. Zorzi, "Idle-time energy savings through wake-up modes in underwater acoustic networks”. Ad Hoc Networks, 2009, vol. 7, no 4, p. 770-777.

[49] D. L. Codiga, J. A., Rice and, P. A., Baxley, "Networked acoustic modems for real-time data delivery from distributed subsurface instruments in the coastal ocean: Initial system development and performance", Journal of Atmospheric and Oceanic Technology, vol. 21, issue 2, pp. 331-346, Feb. 2004

[50] R. M. Eustice, L. L. Whitcomb, H. Singh and, M. Grund, "Experimental results in synchronous-clock one-way-travel-time acoustic navigation for autonomous underwater vehicles", In Robotics and Automation, 2007 IEEE International Conference on, pp. 42574264, April 2007, Roma (Italy)

[51] E. R. B. Marques, M. Pinto, S. Kragelund, P. S. Dias, L. Madureira, A. Sousa, M. Correia, H. Ferreira, R. Goncalves, R. Martins, D. P. Horner, A. J. Healey, G. M. Goncalves and, J. B. Sousa, "AUV control and communication using underwater acoustic networks", Calhoun, 2007. Available at: http://hdl.handle.net/10945/40358 [Last access: March 31, 2015]

[52] A. Bahr, J. J. Leonard and, M. F. Fallon, "Cooperative localization for autonomous underwater vehicles", The International Journal of Robotics Research, vol. 28, issue 6, pp. 714-728, June 2009

[53] A. F. Harris III and, M. Zorzi. "Modeling the underwater acoustic channel in ns2". In Proceedings of the 2nd international conference on Performance evaluation methodologies and tools, October 22 - 26, 2007. Nantes, France (p. 18).

[54] C. Petrioli, R. Petroccia, J. Shusta and, L. Freitag, "From underwater simulation to at-sea testing using the ns-2 network simulator". OCEANS, 2011. June 6-9, 2011, Santander, Spain. (pp. 1-9)

[55] F. Guerra, P. Casari and, M. Zorzi, "World Ocean Simulation System (WOSS): a simulation tool for underwater networks with realistic propagation modeling”. In Proceedings of the Fourth ACM International Workshop on UnderWater Networks. November 04 - 06, 2009, Berkeley, CA, USA. (p. 4-12).

[56] P. Xie, Z. Zhou, Z. Peng, H. Yan, T. Hu, J.H. Cui, Z. Shi, Y. Fei and, S. Zhou, "Aqua-Sim: an NS-2 based simulator for underwater sensor networks". In proceedings of the OCEANS 2009, MTS/IEEE BiloxiMarine Technology for Our Future: Global and Local Challenges. October 26-29, 2009. Biloxi, Mississippi, USA, (pp. 1-7).

[57] S. Sendra, J. Lloret, J., Rodrigues and J. M. Aguiar, "Underwater wireless communications in freshwater at 2.4 GHz". IEEE Communications Letters, 2013, Vol. 17, No.9, pp.1794-1797.

[58] J. Lloret, S. Sendra, M. Ardid and, J. J. P. C. Rodrigues, "Underwater Wireless Sensor Communications at $2.4 \mathrm{GHz}$ ISM Frequency Band”, Sensors, 2012, Vol. 12, No. 4, pp. 4237-4264. 